\title{
ORIGINAL
}

\section{VARIABILIDAD EN VIZCAYA DEL REGISTRO DE LAS ACTIVIDADES PREVENTIVAS EN LA MUJER}

\author{
Irma Múgica del Campo (1), Félix Ibáñez Pérez (1) y Raquel Cobos Campos (2).
}

(1) Centro de Salud Rekalde, Comarca Bilbao. Vizcaya.

(2) Unidad de Investigación de Álava. Hospital Universitario Araba. (Sede Txagorritxu). Vitoria-Gasteiz. Álava

Los autores declaran no tener conflicto de intereses en la realización del estudio.

\section{RESUMEN}

Fundamentos: Diferentes estudios han puesto de manifiesto que existe un déficit de realización de actividades preventivas en nuestro medio, sin embargo, en Vizcaya la situación todavía no se conoce. El objetivo del trabajo es determinar el porcentaje de registro en la historia clínica informatizada (Osabide) de las actividades preventivas realizadas en la mujer por los médicos/as de atención primaria, y analizar las características que condicionan las diferencias en el registro.

Métodos: Estudio descriptivo transversal que incluyó a 425 mujeres con edades comprendidas entre 26 y 65 años que habían acudido a consulta en más de una ocasión y que lo hicieron nuevamente los días 28, 29 ó 30 de abril de 2010. Se recogió como variable principal el registro de prácticas anticonceptivas (grupo de 26 a 51 años), de citologías cervicales durante los últimos 5 años, y de mamografías los últimos 2 años (grupo de 52 a 65 años), y se comparó dicho registro entre médicos y médicas, entre comarcas sanitarias y entre zonas con CAM (Centro de Atención a la Mujer) y sin él, mediante el estadístico chi².

Resultados: Del total de mujeres incluidas en el estudio, en 139 $(34,1 \%)$ existía registro de citología en la historia clínica. De aquéllas con edad entre 26 a 51 años, $99(48,3 \%)$ tenían registro de prácticas anticonceptivas. $22(10,7 \%)$ de las mujeres con edad entre 52 a 65 años tenían registro de mamografia.

Conclusiones: El registro de actividades preventivas en la mujer en Osabide-Vizcaya es bajo. Se han observado diferencias en el registro según sexo del médico.

Palabras clave: Salud pública. Promoción de la salud. Mujeres. Sistemas de registro. Desigualdades en salud, Atención primaria de la salud.

Correspondencia

Raquel Cobos Campos.

Unidad de Investigación de Alava.

Hospital Universitario Araba (sede Txagorritxu).C/José Achótegui s/n 01009-Vitoria-Gasteiz.

raquel.coboscampos@osakidetza.net
ABSTRACT

\section{Registry of Preventive Activities in Women. Variability in Vizcaya, Spain}

Background: Several studies have reported that there is a lack of preventive activities performance in Primary Health Care, however in Vizcaya, the situation is not known, so, we decided determine the percentage of preventive activities carried out on women by primary care physicians which are recorded in the Electronic Health Record (Osabide), and analyze the characteristics which determine differences in registration between regions and physicians.

Methods: We conducted a cross-sectional study involving 425 women aged between 26 and 51 years who were to the general practitioners' consult at least twice, and who were again on April 28, 29 or 30, 2011. We colleted as primary variable the registries of contraceptives practices (group aged between 26 and 51 years), the registries of cervical cytologies during the last five years, as well as the registries of mammograms performed during the two last years, and compared the medical record between men and women practitioners, health regions and between areas with and without centers for women, using the chi-square statistic.

Results: In 139 (34.1\%) of all women and in 99 (48.3\%) of women aged between 26 and 51 years there was registry of cytologies and contraceptives practices respectively. The mammography performance was registered in $22(10.7 \%)$ of women aged between 52 and 65 years.

Conclusions: The registry of preventive activities on women is low. We have observed differences by practitioners' gender.

Key words: Public health. Health promotion. Group women's. Registries. Healthcare disparities. Primary health care. 


\section{INTRODUCCIÓN}

Entre las actividades asistenciales preventivas propias de cualquier Equipo de Atención Primaria (EAP), se encuentran la atención a la mujer adolescente y joven en cuanto a consejos y prescripción de anticoncepción, y en cuanto a actividades preventivas relacionadas con el cáncer ginecológico ${ }^{1,2}$.

Hasta el momento del estudio existía evidencia, aunque controvertida, sobre el beneficio del cribado poblacional del cáncer de mama (CM). A pesar de la polémica, los programas de detección precoz, junto con los avances diagnósticos y terapéuticos, han demostrado contribuir al aumento de la supervivencia, por encima del $75 \%$ a los 5 años del diagnóstico ${ }^{3,4}$.

El cáncer de cervix (CC) tiene baja incidencia y baja mortalidad en España, por debajo de la media europea, pero reviste especial importancia por afectar a mujeres jóvenes. Las citologías periódicas se consideran el mejor método disponible para la prevención de la enfermedad, con una disminución de la incidencia de hasta el $80 \%{ }^{5,6}$.

Por otra parte, la tasa de abortos en España y en la Comunidad Autónoma del País Vasco (CPAV) viene experimentando un crecimiento constante ${ }^{7}$, sin embargo, la población femenina no accede en general a los sanitarios de atención primaria (AP) como primer recurso para la prevención del embarazo no deseado.

Por circunstancias políticas y legales, durante los años de la transición democrática, una parte de esta demanda asistencial (la relacionada con la anticoncepción) fue cubierta en buena parte por profesionales socio-sanitarios apoyados por asociaciones vecinales en Centros de Atención a la Mujer (CAM), pero pocos años después, primero en ambulatorios y luego en centros de salud (CS), los médicos/as de familia (MF) fueron consolidando paulatinamente un estilo asistencial propio, en el que las actividades preventivas serían un hecho diferenciador con la actividad del anterior "médico/a de cabecera".

A pesar de ello, después de tanto tiempo seguimos pensando que existe un déficit en la realización de estas actividades en nuestro medio, que ha sido a su vez puesto de manifiesto en diferentes estudios realizados en otra comunidades autónomas ${ }^{7,8}$ y además éste se ve acentuado por la deficiente explotación de la Historia Clínica Informatizada (Osabide) en algunas comarcas y, especialmente, por la ausencia de coordinación con los otros niveles implicados: CAM, atención especializada (AE) y programas específicos de cribado como el Programa de Diagnóstico Precoz del Cáncer de Mama (PDPCM).

El objetivo del trabajo es determinar el porcentaje de registro en la historia clínica informatizada (Osabide) de las actividades preventivas realizadas en la mujer por los médicos/as de atención primaria y analizar las características que condicionan sus diferencias en el registro.

\section{SUJETOS Y MÉTODOS}

Diseño del estudio. Estudio descriptivo transversal en el que participaron 30 MF de 8 CS diferentes de las 4 comarcas sanitarias de Vizcaya (2 centros por comarca): Bilbao, Ezkerraldea, Interior y Uribe.

Criterios de inclusión y exclusión: Se incluyeron en el estudio mujeres con edades comprendidas entre los 26 y 65 años que habían acudido a consulta en más de una ocasión y que lo hicieron nuevamente los días 28,29 ó 30 de abril de 2010. La única causa de exclusión fue 
la histerectomía y la ausencia de relaciones sexuales coitales en mujeres de 2651 años. Se invitó a participar a todos los médicos/as de atención primaria de Vizcaya que previamente habían manifestado su interés por el tema a estudio. El método empleado para solicitar la colaboración de los médicos/as fue el correo electrónico. Tras haber manifestado su deseo de participar en el estudio, se concertó una entrevista personal con cada uno de ellos/as y se procedió a la revisión de las historias clínicas (HC) conjuntamente.

Variables: Se recogieron datos acerca del registro de prácticas anticonceptivas (grupo de 26 a 51 años) y de citologías cervicales durante los últimos 5 años, así como de mamografías (grupo de 52 a 65 años) durante los últimos 2 años.

Análisis estadístico: Se realizó una descripción de las características generales de la muestra. Las variables cuantitativas se expresaron con la media y la desviación estándar y las cualitativas con el porcentaje y el intervalo de confianza al 95\%. Se comparó el registro de prácticas anticonceptivas y citologías en los últimos cinco años y mamografía en los últimos dos años entre médicos y médicas, entre comarcas sanitarias y entre aquellas zonas con CAM y sin él, mediante la prueba de $\mathrm{chi}^{2}$. Posteriormente se realizó un análisis multivariante para cada una de las variables (registro de prescripción anticonceptiva, mamografías y citologías) en la que se tuvieron en cuenta todas las posibles variables confundidoras (sexo del médico, comarca y existencia o no de CAM en la zona de influencia del CS). Se consideró un resultado estadísticamente significativo cuando el valor de $\mathrm{p}$ fue inferior a 0,05 . Los resultados fueron analizados con el programa estadístico IBM SPSS STATISTICS versión 18.0.

\section{RESULTADOS}

\section{Características generales de la mues-}

tra: El número total de mujeres finalmente incluidas en el estudio fue de 425 ( 6 fueron excluidas antes del inicio): 208 en el grupo de edad de $26-51$ años $(48,9 \%)$ y 217 en el grupo de 52-65 años (51,1\%). La edad media de la muestra fue $49,3 \pm 11,8$ años.

Del total de mujeres incluidas en el estudio, en 139 (34,1\%) se encontró información sobre la realización de citologías en la historia clínica. De entre las mujeres con edad comprendida entre 26 a 51 años, 99 $(48,3 \%)$ tenían información acerca de prácticas anticonceptivas. 22 (10,7\%) mujeres de las del grupo de 52 a 65 años, tenían registro de mamografía.

Asociación entre registro de prácticas preventivas y sexo del médico, comarca sanitaria y existencia o no de CAM. Al realizar el análisis univariante se observó que las médicas realizaron un mayor registro de prácticas anticonceptivas y de citologías que los médicos [54 $(63,5 \%)$ frente a $45(37,5 \%)(\mathrm{p}<0,001)$ y $80(47,1 \%)$ frente a $59(24,8 \%) ; \mathrm{p}<0,001$ respectivamente] (tabla 1).

No se observaron diferencias estadísticamente significativas en el registro de mamografía entre la comarca de Bilbao y el resto (Uribe, Interior y Ezkerraldea) $(p=0,092)$.

Se observó que el hecho de tener como referencia un CAM estaba relacionado con un menor registro de prácticas anticonceptivas [31 $(34,8 \%)(\mathrm{p}=0,001)]$, citologías [17 $(9,9 \%)(\mathrm{p}<0,001)]$ y mamografía [3 $(3,7 \%), p=0,008]$.

Ya que en el análisis univariante se observó una relación positiva entre el hecho de que no exista CAM en la zona de salud y un mayor registro por parte de los 


\begin{tabular}{|c|c|c|c|c|c|c|c|c|}
\hline \multirow{13}{*}{ 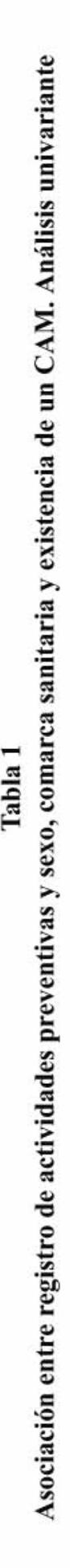 } & \multirow{2}{*}{\multicolumn{2}{|c|}{$\begin{array}{l}0 \\
\sum \\
\sum\end{array}$}} & \multicolumn{2}{|c|}{$\begin{array}{l}\overrightarrow{8} \\
0\end{array}$} & \multicolumn{2}{|c|}{$\begin{array}{l}\overline{8} \\
\dot{8} \\
\dot{v}\end{array}$} & \multicolumn{2}{|c|}{$\begin{array}{l}\infty \\
0 \\
0 \\
0\end{array}$} \\
\hline & & & 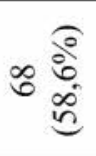 & 疋 & 줄 & 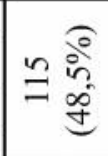 & 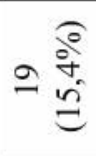 & 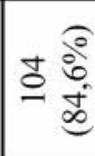 \\
\hline & 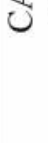 & in & 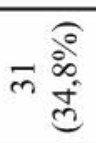 & 闹 & $=\begin{array}{l}\hat{\circ} \\
\hat{\sigma}\end{array}$ & ㅎํㅇ & शे & 20 \\
\hline & & D. & \multicolumn{2}{|c|}{$\begin{array}{l}\overline{8} \\
8 \\
8\end{array}$} & \multicolumn{2}{|c|}{$\begin{array}{l}\overline{8} \\
8 \\
0\end{array}$} & \multicolumn{2}{|c|}{$\stackrel{\text { Oे }}{\circ}$} \\
\hline & \multirow{4}{*}{ 选 } & 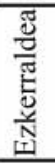 & ㅇํํ & 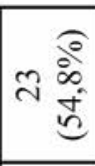 & m $\frac{\text { ণิ }}{ \pm}$ & $\stackrel{\substack{0 \\
\infty}}{\substack{\infty \\
\infty}}$ & $n \stackrel{8}{0}$ & 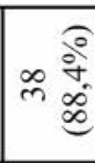 \\
\hline & & 泀 & ๙ู & $\stackrel{\frac{8}{2}}{\frac{0}{2}}$ & 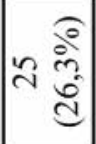 & $\stackrel{\overparen{2}}{\stackrel{2}{*}}$ & $\nabla \stackrel{\text { 요 }}{\infty}$ & $\stackrel{\frac{\hat{\rho}}{\sigma}}{\stackrel{\infty}{a}}$ \\
\hline & & 莣 & क & $\pm \frac{8}{2}$ & $=\begin{array}{l}\hat{0} \\
\hat{j} \\
\hat{g}\end{array}$ & m & 의 $\stackrel{\text { กे }}{\text { a }}$ & 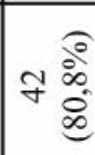 \\
\hline & & $\begin{array}{l}\stackrel{\mathscr{J}}{0} \\
\stackrel{0}{=0}\end{array}$ & 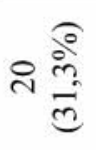 & $\forall \stackrel{\substack{\infty \\
\infty}}{\infty}$ & $\infty \begin{array}{l}\text { हैं } \\
\text { î }\end{array}$ & 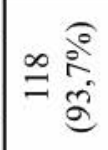 & 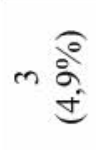 & 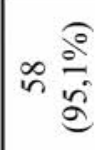 \\
\hline & & $a$ & \multicolumn{2}{|c|}{$\begin{array}{l}\overline{8} \\
\dot{0} \\
\dot{1}\end{array}$} & \multicolumn{2}{|c|}{$\begin{array}{l}\overline{8} \\
8 \\
\overline{8}\end{array}$} & \multicolumn{2}{|c|}{$\frac{n}{0}$} \\
\hline & \multirow{2}{*}{ 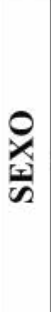 } & 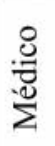 & 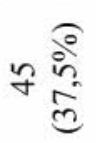 & ^ & 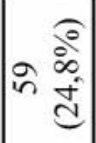 & פ & $a \stackrel{2}{2}$ & 尺̊̊ \\
\hline & & 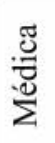 & ॠ & क & 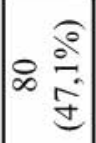 & ๙̊ํํ & 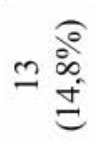 & ๙ \\
\hline & & & $\vec{n}$ & @ & $\bar{w}$ & 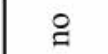 & $\bar{w}$ & @ \\
\hline & & & \multicolumn{2}{|c|}{ 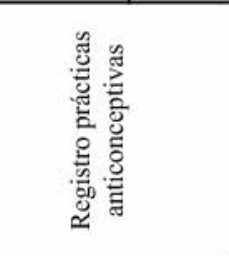 } & \multicolumn{2}{|c|}{ 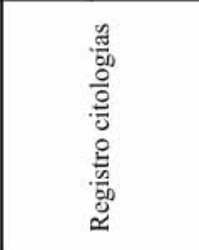 } & \multicolumn{2}{|c|}{ 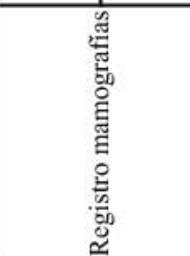 } \\
\hline
\end{tabular}


Tabla 2

Asociación entre registro de actividades anticonceptivas y sexo del médico, comarca sanitaria y CAM. Análisis multivariante

\begin{tabular}{|c|c|c|c|c|c|c|}
\hline & & \multicolumn{5}{|c|}{ Registro de prácticas anticonceptivas } \\
\hline & & $\mathrm{NO}$ & SI & $\mathrm{p}$ & $\mathrm{OR}$ & IC $95 \% * *$ \\
\hline \multirow{2}{*}{ Sexo } & Médico & $75(62,5 \%)$ & $45(37,5 \%)$ & & & \\
\hline & Médica & $31(36,5 \%)$ & $54(63,5 \%)$ & 0,017 & 2,23 & $1,154-4,308$ \\
\hline \multirow{4}{*}{ Comarca } & Bilbao & $44(68,8 \%)$ & $20(31,3 \%)$ & 0,201 & & \\
\hline & \begin{tabular}{|l} 
Uribe \\
\end{tabular} & $14(27,5 \%)$ & $37(72,5 \%)$ & 0,052 & 3,673 & $0,991-13,615$ \\
\hline & Interior & $25(52,1 \%)$ & $23(47,9 \%)$ & 0,206 & 1,771 & $0,730-4,294$ \\
\hline & Ezkerraldea & $23(54,8 \%)$ & $19(45,2 \%)$ & 0,374 & 1,849 & $0,478-7,160$ \\
\hline \multirow{2}{*}{ CAM* } & \begin{tabular}{|l|}
$\mathrm{Si}$ \\
\end{tabular} & $58(65,2 \%)$ & $31(34,8 \%)$ & & & \\
\hline & No & $48(41,4 \%)$ & $68(58,6 \%)$ & 0,74 & 1,201 & 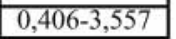 \\
\hline
\end{tabular}

* Centro de Atención a la Mujer;**IC 95\%: Intervalo de confianza al 95\%

Tabla 3

Asociación entre registro de citologías y sexo del médico, comarca sanitaria y CAM. Análisis multivariante

\begin{tabular}{|c|c|c|c|c|c|c|}
\hline & & \multicolumn{5}{|c|}{ Registro de citologias } \\
\hline & & $\mathrm{NO}$ & SI & $\mathrm{p}$ & OR & IC $95 \% \%^{* *}$ \\
\hline \multirow{2}{*}{ Sexo } & Médico & $179(75,2 \%)$ & $59(24,8 \%)$ & & & \\
\hline & Médica & $90(52,9 \%)$ & $80(47,1 \%)$ & 0,021 & 1,913 & $1,104-3,315$ \\
\hline \multirow{4}{*}{ Comarca } & Bilbao & $118(93,7 \%)$ & $8(6,3 \%)$ & $<0,001$ & & \\
\hline & Uribe & $31(30,4 \%)$ & $71(69,6 \%)$ & $<0,001$ & 13,795 & $4,422-43,034$ \\
\hline & Interior & $70(73,7 \%)$ & $25(26,3 \%)$ & 0,006 & 3,677 & $1,445-9,357$ \\
\hline & \begin{tabular}{|l} 
Ezkerraldea \\
\end{tabular} & $50(58,8 \%)$ & $35(41,2 \%)$ & 0,002 & 6,14 & $1,896-19,881$ \\
\hline \multirow{2}{*}{$\mathrm{CAM}^{*}$} & $\mathrm{Si}$ & $154(90,1 \%)$ & $17(9,9 \%)$ & & & \\
\hline & No & $115(48,5 \%)$ & $122(51,5 \%)$ & 0,092 & 2,135 & $0,884-5,157$ \\
\hline
\end{tabular}

* Centro de Atención a la Mujer; ${ }^{* *}$ IC 95\%: Intervalo de confianza al 95\%

Tabla 4

Asociación entre registro de mamografías y sexo del médico, comarca sanitaria y CAM. Análisis multivariante

\begin{tabular}{|c|c|c|c|c|c|c|}
\hline & \multicolumn{5}{|c|}{ Registro de mamografías } \\
\hline & & NO & SI & $\mathbf{p}$ & $\overline{\text { OR }}$ & IC $95 \%$ क* \\
\hline \multirow{2}{*}{ Sexo } & Médico & $108(92,3 \%)$ & $9(7,7 \%)$ & & & \\
\hline & Médica & $75(62,5 \%)$ & $13(14,8 \%)$ & 0,386 & 1,599 & $0,553-4,622$ \\
\hline \multirow{4}{*}{ Comarca } & Bilbao & $58(31,7 \%)$ & $3(13,6 \%)$ & 0,965 & & \\
\hline & Uribe & $42(23,0 \%)$ & $10(45,5 \%)$ & 0,909 & 1,12 & $0,161-7,808$ \\
\hline & Interior & $45(24,6 \%)$ & $4(18,2 \%)$ & 0,881 & 0,871 & $0,143-5,306$ \\
\hline & Ezkerraldea & $38(20,8 \%)$ & $5(22,7 \%)$ & 0,847 & 0,823 & $0,114-5,939$ \\
\hline \multirow{2}{*}{ CAM } & Si & $79(96,3 \%)$ & $3(3,7 \%)$ & & & \\
\hline & No & $104(84,6 \%)$ & $19(15,4 \%)$ & 0,101 & 4,438 & $0,748-26,32$ \\
\hline
\end{tabular}

* Centro de Atención a la Mujer;**IC 95\%: Intervalo de confianza al 95\% 
médicos/as de las tres actividades preventivas (prácticas anticonceptivas, citologías y mamografías), se realizó un análisis multivariante para comprobar si realmente esta diferencia era propiciada por la existencia o no de CAM en la zona de residencia o podía verse afectado por otros factores tales como el sexo del médico o la comarca sanitaria (tablas 2, 3 y 4).

Tras ajustar por sexo y comarca, ya no se observó diferencia estadísticamente significativa en el registro de las actividades preventivas entre las zonas con CAM frente a las que no tienen CAM, por lo que las variables "sexo del facultativo" y "comarca sanitaria" son variables confundidoras que pueden propiciar la aparición de una asociación que en realidad no existe, o que no es de la magnitud que parece (registro de prácticas anticonceptivas $\mathrm{p}=0,740$; registro de citologías $\mathrm{p}=0,092$; registro de mamografías $\mathrm{p}=0,101$ ).

Sin embargo, tras ajustar por comarca y CAM sí que se observó diferencia estadísticamente significativa en el registro de prácticas anticonceptivas y citologías entre ambos sexos $(p=0,017$; OR 2,230 y $\mathrm{p}=0,021$; OR 1,913 , respectivamente), por lo que dicha relación no se vio modificada por ninguna de las dos variables restantes (tablas 2 y 3) y fue la variable sexo por sí sola la que determinó el mayor o menor registro de las actividades preventivas. En cuanto a la "comarca" la relación con el registro de prácticas preventivas varió más, ya que tras ajustar por sexo y CAM únicamente se observó diferencia estadísticamente significativa entre comarcas en el registro de citologías (tabla 3 ).

\section{DISCUSIÓN}

Del total de $\mathrm{HC}$ revisadas en las mujeres con edades comprendidas entre 26 y 51 años únicamente constaba registro sobre la práctica del tratamiento anticonceptivo en algo menos de la mitad. Consideramos que es una cifra baja, indicativa de la escasa capacidad que tenemos para conocer el riesgo de embarazo no deseado y de infecciones de transmisión sexual y promover relaciones sexuales satisfactorias. Sin embargo estos datos han de ser tomados con cierta cautela, ya que se excluyó al segmento de población con mayores tasas de interrupciones voluntarias del embarazo (IVE) (mujeres de 20 a 24 años con 19,82 IVE por 1.000 habitantes en el año 2010). Sería conveniente evaluar el registro de prácticas anticonceptivas en este grupo de edad y promover prácticas preventivas, ya que únicamente el $54 \%$ de las mujeres de entre 16 y 24 años usa métodos anticonceptivos, observándose una mayor frecuencia en las clases sociales más favorecidas $^{9-11}$.

A partir de los resultados del análisis multivariante del registro de actividades anticonceptivas por comarca, parece ser que pertenecer a una $u$ otra comarca sanitaria no influye en el mayor o menor registro de actividades anticonceptivas.

En cuanto al despistaje del carcinoma de cuello uterino, a pesar de la baja incidencia en nuestro país, hay que remarcar que su cribado tiene especial trascendencia, ya que la mayoría podría ser diagnosticado en estadíos precancerosos y además es una patología que afecta especialmente a mujeres jóvenes entre 35-50 años.

En nuestro estudio el $34,1 \%$ del total de mujeres tenía registrado el resultado de una citología cervical en los últimos cinco años. Nuestros datos, aunque no exactamente comparables por la distribución de los grupos de edad, están por debajo de los citados tanto en la Encuesta Nacional de Salud del 2006 ${ }^{12}$, la Encuesta Europea de Salud (datos de España) del $2009^{13}$, y la Encuesta de Salud de la CAV (ESCAV) del $2007^{14}$, en las que el 71,5\% de las mujeres de entre 20-64 años decían haberse realizado una citología en los últimos tres años y un $17,2 \%$ nunca $^{12-14}$. La diferencia en el registro de citologías entre comarcas es 
muy llamativa y de nuevo destacan negativamente los resultados de Bilbao con tan sólo $6,3 \%$ registros en Osabide-Vizcaya frente a $46,45 \%$ en el resto de las comarcas. Además, tras ajustar por sexo y CAM, esta diferencia no desaparece, por lo que el hecho de pertenecer a una u otra comarca parece influir en el mayor o menor registro de citologías. En la comarca de Bilbao no disponemos en la $\mathrm{HC}$ de un formulario específico para esta actividad preventiva ni de un protocolo de coordinación con otros profesionales implicados, existentes en otras comarcas y causa fundamental, creemos, para estos malos resultados.

El cáncer de mama es la primera causa de muerte en mujeres en España y el tumor más frecuente en las mujeres occidentales. La detección poblacional del cáncer de mama es un servicio que desde el año 1990 se ha ido incluyendo en la oferta de servicios de las CCAA. El PAPPS 6 y la mayoría de sociedades científicas y organismos oficiales recomiendan en mujeres de bajo riesgo la mamografía bienal a partir de los 50 años. En nuestro estudio destaca notablemente el infrarregistro de la realización de mamografía en los dos últimos años: sólo aparece en el 10,7\% de las $\mathrm{HC}$ de mujeres de entre 52-65 años. Este dato contrasta con el del PDPCM de junio de 2010 de nuestra comunidad autónoma en mujeres de entre 50 y 64 años, con una implantación del $80,6 \%{ }^{15}$, y con las cifras de la Encuesta de Salud de la CAPV del año 2007 (ESCAV) en la que el 91,1\% de las mujeres de ese tramo de edad referían haberse realizado una mamografía en los últimos 3 años ${ }^{14}$.

Los bajos resultados en todas las comarcas son explicables por la uniformidad del PDPCM de Vizcaya, pero denotan la falta de coordinación con atención especializada, ya que en ninguna de las cuatro comarcas se registra apenas dicha actividad.

En general, se ha observado un bajo registro en Osabide-Vizcaya en las tres actividades preventivas evaluadas, resultados que coinciden con los obtenidos por otros investigadores/as en otras comunidades autónomas ${ }^{7,8}$.

Aunque en la actualidad todos los expertos/as están convencidos de la necesidad de la integración de las actividades preventivas en la actividad asistencial, la realidad es que se practica muy poco la medicina preventiva. La mayoría de los esfuerzos y del tiempo son dedicados al diagnóstico y tratamiento de las enfermedades y muy poco a la prevención ${ }^{16}$. Esto podría ser debido a varios factores, tales como falta de motivación de los profesionales y tiempo en la consulta, escasa formación continuada $y$, en consecuencia, desconocimiento de las medidas preventivas a aplicar ${ }^{16}$. Además, consideramos que se debe analizar la evolución de la práctica preventiva en cada centro a lo largo de los años, pues se produce un estancamiento con el tiempo.

En nuestro estudio también se observó una diferencia estadísticamente significativa en el registro de las tres actividades preventivas entre los cupos atendidos por médicos y médicas. Resultados similares fueron encontrados en un estudio realizado en Andalucía ${ }^{17}$.

La variabilidad clínica entre los profesionales médicos/as en esgeneral alta. Hay una variabilidad necesaria y aceptable, que es la marca de la adaptabilidad a contextos y situaciones complejas, como lo es la consulta diaria del MF, pero en muchas ocasiones esta variabilidad denota un déficit de calidad asistencial y, por buscarle el lado positivo, una gran posibilidad de mejora. Además de los factores dependientes del profesional, en esta variabilidad pueden influir diferencias en los sistemas de salud, carga asistencial, aspectos organizativos, utilización de pautas protocolizadas, siste- 
mas de recordatorio, preferencias del/de la paciente o del/de la profesional cuando existe más de una opción aceptada científicamente, etc. ${ }^{18-20}$. Sin embargo, aunque hay gran variabilidad intra e inter comunidades autónomas, los MF españoles realizamos en mayor porcentaje que nuestros/as colegas europeos/as actividades de promoción y prevención de la salud de mayor evidencia científica ${ }^{21}$.

Para poder limitar la variabilidad clínica en el trabajo diario son fundamentales su monitorización con indicadores de adecuación a los procesos, protocolos y guías clínicas basadas en la evidencia y, por otro lado, la informatización de la historia clínica y la existencia de repositorios documentales y "sistemas inteligentes" que ayuden a la decisión y el seguimiento clínico de los/as pacientes.

La principal limitación de nuestro estudio ha sido la selección de la muestra, ya que únicamente se invitó a participar a los/as médicos /as que previamente habían manifestado interés por el tema que nos ocupa. Esto puede haber provocado un sesgo de selección por haber elegido únicamente a los centros de salud o médicos/as más involucrados/as en este tipo prácticas $\mathrm{y}$, por consiguiente, haber sobreestimado la medición de la variable principal (registro de prácticas preventivas).

Otra limitación ha sido haber excluido a las mujeres con edades entre 16 y 24 años en la medición del registro de prácticas anticonceptivas cuando el grupo con mayores tasas de IVE es el grupo de mujeres de entre 20 a 24 años.

Los Equipos de Atención Primaria tendrían que mejorar el registro de actividades preventivas, pues ésta es una de las funciones que se realiza diariamente en la consulta. Además de este escaso conocimiento se podría deducir una limitada capacidad de intervención y en consecuencia una peor calidad asistencial a la mujer.
La presencia de un CAM en la zona de influencia de los CS no está asociada por sí sola con una menor tasa de registro de actividades preventivas, al contrario de lo que se suponía en un principio. Existen otras variables, como son el sexo del médico y la comarca sanitaria, las que modifican esta asociación por lo que se consideran variables confundidoras.

Creemos que nuestro rendimiento es peor cuando la $\mathrm{HC}$ informatizada no nos facilita o recuerda la pertinencia de este tipo de actividad y cuando no existen protocolos de actuación conjuntos con la atención especializada. En la comarca de Bilbao, la nuestra, al contrario que en las otras tres, se dan estas dos circunstancias, aunque hay que mencionar que existe un proyecto de despliegue progresivo de cribado de cáncer de cérvix que tratará en lo posible de reducir este infrarregistro en relación al resto de comarcas.

Concluyendo, las médicas tienen en nuestro estudio porcentajes de registro de actividades preventivas mayores que los médicos. Además esta asociación no se ve modificada por variables tales como el pertenecer a una comarca sanitaria u otra ni al hecho de que en la zona de residencia exista un CAM, por lo que el sexo de los facultativos marca claramente la diferencia en el registro.el registro de actividades preventivas en la mujer en Osabide-Vizcaya es bajo.

\section{BIBLIOGRAFÍA}

1. Marzo Castillejo M, Bellas Becerro B, Melus Palazón E, Vela Vallespín C, Nuin Villanueva M, Vilarrubi Estrella M. PAPPS actualización 2009. Prevención del cáncer. Disponible en:

http://www.papps.org/upload/file/10\%20PAPPS $\% 2$ 0ACTUALIZACION\%202009.pdf

2. Estudio EPEAS. Estudio sobre la seguridad de los pacientes en el Sistema Nacional de Salud. Madrid: Ministerio de Sanidad y Consumo; 2008. Disponible en: http://www.msc.es/organizacion/sns/planCalidadSNS/docs/Modulo3.pdf. 
3. Nelson HD, Tyne K, Naik A, Bougatsos C, Chan B Humphrey L. Screening for Breast Cancer: An Update for the U.S. Preventive Services Task Force. Ann Intern Med. 2009;151:727-37.

4. Lee CH, Dershaw DD, Kopans D, Evans P, Monsees B, Monticciolo D, et al. Breast Cancer Screening With Imaging: Recommendations From the Society of Breast Imaging and the ACR on the Use of Mammography, Breast MRI, Breast Ultrasound, and Other Technologies for the Detection of Clinically Occult Breast Cancer. Am Coll Radiol. 2010;7:18-27.

5. Marzo M, Cierco P, del Cura I. Prevención del cáncer de cérvix. Aten Primaria. 2005;6:328-33.

6. Cabanes-Domenech A, Pérez-Gómez B, Aragonés N, Pollán M, López-AbenteG. La situación del cáncer en España. 1975-2006. Madrid: Centro Nacional de Epidemiología. ISCIII; 2009. Disponible en: http://www.isciii.es/htdocs/pdf/epicancerjunio2009.pdf.

7. Vives-Argilagós A, Ballvé-Moreno JL, BerenguéIglesias MD, Monteverde-Curto X, Calero-Muñoz C, Cierco-Peguera $\mathrm{P}$, et al. Validez del registro de las actividades preventivas en las historias clínicas: consumo y consejo antitabaco. Aten Primaria. 1996; 18: 309-14.

8. Fernandez-Vergel, R, Gadea-Font M, SanjuánSanchís E, Bonich-Juan R, Parellada-Squiss N, Villafáfila-Ferrero R. Actividades preventivas y esquizofrenia: ¿Lo hacemos bien? Medifam. 2001;11(8):3545 .

9. Antona A, Madrid J. Anticoncepción de emergencia, adolescencia y representaciones sociales. Contraception of emergency, adolescente and social perspective. Sexología Integral. 2009; (3): 102-108.

10. Interrupción voluntaria del embarazo y métodos anticonceptivos en jóvenes. Madrid: Ministerio de Sanidad y Consumo. Observatorio de salud de la mujer; 2004. Citado el 4 de mayo de 2012.

11. La interrupción voluntaria del embarazo y los métodos anticonceptivos en jóvenes. Madrid: Ministerio de Sanidad Política Social e Igualdad. Disponible en: http://www.msc.es/profesionales/saludPublica/prevPromocion/embarazo/docs/IVE_2010.pdf. Citado el 4 de mayo de 2012.

12. Encuesta Nacional de salud 2006. Madrid: Ministerio de Sanidad y Política Social. Disponible en: http://www.msps.es/estadEstudios/estadisticas/encu estaNacional/encuestaNac2006/EstilosVidaPorcentaje.pdf.
13. Encuesta Europea de Salud en España 2009. Madrid: Ministerio de Sanidad y Política Social; 2009. Disponible en:

http://www.ine.es/jaxi/menu.do?type=pcaxis\&path= $\% 2 \mathrm{Ft} 15 / \mathrm{p} 420 \&$ file $=$ inebase $\& \mathrm{~L}=0$.

14. Encuesta de Salud de la CAPV 2007. Vitoria: Departamento de Sanidad y Consumo del Gobierno Vasco. Disponible en:

http://www.osanet.euskadi.net/r85escav/es/contenidos/informacion/encuesta_salud_comarcas/es_escav /adjuntos/practicas.pdf.

15. Programa de detección precoz del cáncer de mama en la CAPV. Memoria 2009. Vitoria: Departamento de Sanidad y Consumo del Gobierno Vasco. Disponible en: https://www6.euskadi.net/v19. osk0004/es/contenidos/informacion/memoria09/es memoria/especializada.html\#mama.

16. Salleras L1. Llibre Blanc. Bases per a la integració de la prevenció a la pràctica assistencial. Generalitat de Catalunya, Departament de Sanitat i Seguretat Social. Barcelona: Ediciones Doyma; 1993.

17. Delgado A, López-Fernández LA, Luna JD. Ser médico o médica marca diferencias en la práctica asistencial. Aten Primaria. 2001;27:219-26.

18. Ojeda-Pérez F. Variabilidad clínica. Una visión del profesional médico. Rev Cal Asist. 2006;21:63-5.

19. Turubian JL, Pérez B. La variabilidad es un indicador de buena gestión clínica en medicina de familia. Aten primaria. 2006;37:160-3.

20. Fusté J, Rué M. Variabilidad en las actividades preventivas en los equipos de atención primaria de Cataluña. Aplicación del análisis de niveles múltiples. Gac Sanit. 2001;15:118-27.

21. Kloppe P, Brotons C, Anton JJ, Ciurana R, Iglesias M, Piñeiro R, et al. Prevención y promoción de la salud en atención primaria: comparación entre la visión de los médicos/as españoles y los médicos/as europeos. Aten Primaria. 2005;36:144-51. 